\title{
THE GENETIC BASIS OF "DUALITY" IN IMPERFECT FUNGI
}

\author{
J. L. JINKS \\ A.R.C. Unit of Biometrical Genetics, Department of Genetics, \\ The University, Birmingham
}

Received I.viii.59

The cytoplasmic basis of a heritable difference in phenotype is inferred from its failure to be transmitted from parent to offspring in the manner expected of a nuclear controlled difference. This generally means nonMendelian inheritance, of which reciprocal differences are the simplest evidence. In some fungi reciprocal crosses have been used to distinguish between differences of nuclear and cytoplasmic origin (Mitchell and Mitchell, I952 ; Day, I959) but in most species the life cycle makes it necessary to draw this distinction without the aid of either reciprocal crosses or even of sexual reproduction. In these species we must rely on expectations derived from other properties of nuclear controlled differences to effect this separation.

In heterokaryons the component nuclei sort out as units in the production of asexual spores without any genic recombination between them. Hence each kind of nucleus can be marked and followed unambiguously in asexual spore production by a single mutant gene. If, therefore, in the asexual progeny of a heterokaryon a difference in phenotype fails to remain in association with the nuclear marker with which it entered the heterokaryon then the difference must be under cytoplasmic control (Jinks, I954, I956, 1958, I959; Pittenger, I956 ; Gowdridge, I956 ; Arlett, I957 ; Gibson and Griffin, I958; Mahony and Wilkie, I958; Roper, I958 ; Sharpe, 1958). A single difference in phenotype between two homokaryon isolates cannot be classified directly by this test. Nor can the test be successfully used with two independently occurring differences in phenotype between the components of the heterokaryon unless one is known to be nuclear in origin. However, additional expectations for nuclear controlled differences can be derived frcm cytological investigations. Cytology can trace back the history of the nuclei of the asexual spores of a heterokaryon and tell us whether to expect only homokaryotic spores, or if heterokaryotic spores can be produced, their maximum frequency. It can further tell us whether all the spores in the same chain or head should have identical genotypes (Baker, I944; Pontecorvo and Gemmell, I944; Baker, I945; Miller, I946 ; Rees and Jinks, I952; Pontecorvo, I953 ; Buxton, I954; Ishitani and Sakaguchi, I $955 a, b$; Gibson and Griffin, I 958). Hence, failure of a difference in phenotype to behave according to these expectations would rule out a nuclear origin. On the other hand, agreement with these expectations is not in all cases unambiguous evidence of a nuclear origin.

In sexual species cytoplasmic differences are known which segregate at asexual spore formation in a manner analogous to the sorting out of whole nuclei. This leads to a segregation pattern in the asexual progeny which is similar to that of a heterokaryon producing a high proportion of heterokaryotic spores (Pittenger, I956 ; Arlett, I957 ; Catcheside, I958 ; Gibson and Griffin, I958 ; Jinks, I958, I959). There is no reason to believe that 
similar cytoplasmic differences are not also present in imperfect fungi. Thus in those heterokaryons where we expect the production of a high proportion of heterokaryotic asexual spores, to observe a segregation in the asexual progeny compatible with this expectation is not proof of a nuclear origin.

If we apply these criteria to the many investigations into the nature of the wild variation in the imperfect fungi few can still be regarded as providing conclusive evidence as to its causation. Among those which do are the demonstrations of heterokaryosis in imperfect Penicillium (Jinks, I952a, $b$; Rees and Jinks, I952), Aspergillus (Ishitani and Sakaguchi, I955a, b) and Fusarium (Buxton, I954) species. In these examples the complete accord between the detailed cytological evidence and the segregations observed in the asexual progeny of the heterokaryon leave no doubt as to the nuclear origin of the variation.

The "dual phenomenon" which is probably the most widely reported type of variation in the imperfect fungi has generally been attributed to the nuclei (Hansen and Smith, I932; Hansen, I938, I942; Hansen and Snyder, 1944). The regularity of the segregation of the asexual progenies of "dual "fungi led Hansen to suggest that the "duality" sprang from the persistent association of different nuclei. This explanation has been generally accepted, but its acceptance has rested largely on a correlation claimed between the proportion of asexual spores of a "dual" colony which give rise to "dual" colonies; the number of distinct phenotypes among these "dual" colonies ; and the number of nuclei in the asexual spores. While this correlation may have a general validity such evidence would be more compelling if, as suggested earlier, the origin of the nuclei in the spores was also known. Thus where all the nuclei of a single multinucleate or multicellular spore produced by a heterokaryon arise from the mitotic division of a single nucleus (Baker, I945 ; Buxton, I954; Ishitani and Sakaguchi, 1955 ; Gibson and Griffin, I958) a "duality" based on heterokaryosis can no more be transmitted through single asexual spores than in those species of fungi which produce only uninucleate spores (Baker, I944; Miller, 1946). The evidence, as it stands at present, therefore, by no means rules out a cytoplasmic origin of variation included under the heading of the "dual phenomenon". Furthermore, every characteristic of the "dual phenomenon" can be paralleled by cytoplasmic determinants. The cultural, morphological and physiological differences which distinguish the components of a "dual " fungus can arise by cytoplasmic change. They arise spontaneously especially in aged material (Jinks, I954, I956, I958, I 959 ; Gibson and Griffin, I958 ; Sharpe, I958 ; Marcou and Schecroun, I959), they may be recovered from the wild and they are readily induced by a variety of treatments (Arlett, I957; Jinks, I958 ; Roper, I958).

The changed cytoplasmic determinants are capable of existing side by side with their wild-type, unchanged counterparts (Pittenger, I956 ; Arlett, I957; Jinks, I958, I959; Gibson and Griffin, I958; Marcou and Schecroun, 1959). These mixed cytoplasms or heteroplasmons can be produced in a manner analogous to heterokaryon formation (Gowdridge, I956 ; Pittenger, I956 ; Arlett, I957 ; Gibson and Griffin, I958 ; Jinks, I959; Marcou and Schecroun, 1959) or may arise spontaneously by mutation in some, but not all, of the representatives of a cytoplasmic element within a spore or hypha (Arlett, I957 ; Jinks, I958, I959). 
Segregation from a cytoplasm of mixed origin has been obtained in five species of fungi (Pittenger, I956 ; Arlett, I957 ; Gibson and Griffin, I958 ; Jinks, I958, I959; Marcou and Schecroun, I959) and in others, observations compatible with such a segregation have been reported (Mahony and Wilkie, I958 ; Roper, I958 ; Sharpe, I958). In fact, segregation from a cytoplasm of mixed origin has been used as a critical test for detecting functional differences between the components of the mixture (Arlett, I957; Jinks, I958, I959).

Thus our present knowledge of the behaviour of mixed cytoplasms suggests that they could afford a satisfactory alternative to heterokaryosis as an explanation of the "dual phenomenon".

With this possibility in mind four examples of the "dual phenomenon" in new isolates of Penicillium species of the Assymetrica group have been examined using our criteria for distinguishing between differences of nuclear and cytoplasmic origin in imperfect fungi. A cytological investigation showed that only one nucleus entered each asexual spore. Thus, irrespective of the final number of nuclei per spore they would remain homokaryotic. Hence a cytoplasmic basis of the "duality" was inferred for two of the four isolates when over 500 single spore propagations gave I 7 per cent. "dual" colonies which were capable of further segregation. This was made even clearer when a mutation from green to white spores occurred in one component of one of these two isolates. This mutant segregated exactly as predicted for a nuclear controlled difference in a heterokaryon test with its wild-type partner, giving only white spored and green spored homokaryotic colonies. Furthermore, the spore colour difference segregated independently of the difference in colonial morphology and sporulation density which is the basis of the "duality" in this isolate.

Each of the two remaining isolates gave only two stable phenotypes in their asexual progenies; one with normal sporulation and one which produced abundant mycelium but few spores. One of these isolates was investigated further. A white spore colour variant was induced in its normal sporulating component, and a heterokaryon made between this and its wild-type low sporing partner. In the asexual progeny of the heterokaryon, spore colour and sporulation density remained in their original association. Hence both the initial difference in sporulation density and the difference in spore pigmentation are presumably under nuclear control in this isolate.

Thus in our material the "dual phenomenon" may have either a nuclear or a cytoplasmic basis and hence a "dual" colony may be either a heterokaryon or a heteroplasmon. And while the importance of nuclear variation has long been appreciated, these results suggest that cytoplasmic variation is an equally important but neglected source of variation in wild imperfect fungi.

Acknowledgments.-I am indebted to Professor K. Mather, C.B.E., F.R.s., and to Mr L. G. Wigan for advice and criticism during the preparation of this report.

\section{REFERENCES.}

ARLETT, C. F. 1957. Induction of cytoplasmic mutations in Aspergillus nidulans. Nature, Lond., I79, I $250-125 \mathrm{I}$.

BAKER, G. E. 1944. Heterocaryosis in Penicillium notatum. Bull. Torrey. Bot. Cl., 7I, $367-373$. 
BAKER, G. E. I945. Conidium formation in Aspergillus. Mycologia, 37, $582-600$.

BUXTON, E. W. I954. Heterocaryosis and variability in Fusarium oxysporum f. gladioli. 7. Gen. Microbiol., Io, $7 \mathrm{I}$.

CATCHESIDE, D. G. 1958. A discussion on the cytoplasm in variation and development. Proc. Roy. Soc. B., $148,285-290$.

DAY, P. R. 1959. A cytoplasmically controlled abnormality of the tetrads of Coprinus lagopus. Heredity, 13 $_{3}, 8 \mathrm{I}-87$.

Gibson, A., AND GRIFFIN, D. M. 1958. A study of variation in Nectria stenospora. Aust. 7. Biol. Sci., II, 548-556.

GOWDRIDGE, B. I956. Heterocaryosis between strains of Neurospora crassa with different cytoplasms. Genetics, $4 I, 780-789$.

HANSEN, H. N. 1938. The dual phenomenon in imperfect fungi. Mycologia, 30, $442-455$.

hansen, H. N. 1942. Heterocaryosis and variability. Phytopath., 32, 639-640.

HANSEN, H. N., AND SMITH, R. E. I932. The mechanism of variation in imperfect fungi : Botrytis cinerea. Phytopath., 22, 953-964.

HANSEN, H. N., AND SNYDER, W. C. I944. Relation of dual phenomenon in Penicillium notatum to penicillin production. Science, 99,264 .

ISHITANI, C., AND SAKAGUCHI, K. I955a. Hereditary, variation and genetic recombination in Koji-molds I Natural Variation. 7. Gen. and Appl. Microbiol., I, 246-257.

IsHITANI, C., AND SAKAGUCHI, K. I $955 b$. Hereditary, variation and genetic recombination in Koji-molds IV Cytological studies. 7. Gen. and Appl. Microbiol., I, 283-297.

JiNks, J. L. I952a. Heterocaryosis in wild Penicillium. Heredity, 6, 77.

JINKs, J. L. I952b. Heterocaryosis : a system of adaptation in wild fungi. Proc. Roy. Soc. B., I40, 83-99.

JINKS, J. L. 1954. Somatic selection in fungi. Nature, Lond., I74, 409.

JiNks, J. L. 1956. Naturally occurring cytoplasmic changes in fungi. C. R. Lab. Carlsberg série physiologique, 26, 183-203.

JiNks, J. L. 1958. Cytoplasmic differentiation in fungi. Proc. Roy. Soc. B., I48, 3I4-32I.

JINKS, J. L. I959. Lethal, suppressive cytoplasms in aged clones of Aspergillus glaucus. F. Gen. Microbiol., 21, 397-409.

MAHONY, M., AND WILKIE, D. I958. An instance of cytoplasmic inheritance in Aspergillus nidulans. Proc. Roy. Soc. B., I48, 359-36 I.

marcou, D., AND SGHecroun, J. I959. La sénescence chez Podospora pourrait être due a des particules cytoplasmiques infectantes. $C$. $R$. des seances de l'Acad. de Sci., 248, 280-283.

Miller, J. J. 1946. The theories concerning variability in fungi. Abs. Canad. Phytopath. Soc., 14, I3.

MITCHELL, M. B., AND MITCHELL, H. K. I952. A case of "maternal" inheritance in Neurospora crassa. Proc. Nat. Acad. Sci. Wash., 38, 442-449.

PITTENGER, T. H. I956. Synergism of two cytoplasmically inherited mutants in Neurospora crassa. Proc. Nat. Acad. Sci. Wash., 42, 747-752.

pontegorvo, G. 1953. The genetics of Aspergillus nidulans. Advance. Genet., 5, I4 I-238.

PONTECORVO, G., AND GEMMELL, A. R. I944. Genetic proof of heterocaryosis in Penicillium notatum. Nature, Lond., 154, 532.

REES, H., AND JINKS, J. L. I952. The mechanism of variation in Penicillium heterokaryons. Proc. Roy. Soc. B., I40, I00-106.

ROPER, J. A. 1958. Nucleo-Cytoplasmic interactions in Aspergillus nidulans. Cold. Spr. Hbr. Symp. Quant. Biol., 23, I4I-I 54 .

SHARPE, H. s. 1958. A closed system of cytoplasmic variation in Aspergillus glaucus. Proc. Roy. Soc. B., 148, 355-359. 